\title{
Sound of a nut rolling inside an elastic rubber balloon
}

\author{
Amaury Barral ${ }^{1, *}$, Quentin Louis ${ }^{1, * *}$, Clément Brochet ${ }^{1}$, Julie André ${ }^{1}$, Anthony Guillen ${ }^{1}$, \\ Fang Goh ${ }^{1}$, Angel Prieto ${ }^{1}$, and Thibault Guillet ${ }^{2}$ \\ ${ }^{1}$ Ecole Polytechnique, 91128 Palaiseau Cedex, France \\ ${ }^{2}$ LadHyX, UMR 7646 du CNRS, Ecole Polytechnique, 91128 Palaiseau Cedex, France
}

Received: 4 July 2018 / Accepted: 14 June 2019

\begin{abstract}
This article studies the sound produced by nuts of different shapes swirling in an elastic rubber balloon. First, the sound is studied experimentally, both in terms of frequency and amplitude. Regarding the frequency components of the sound, we show that they can be divided into two main contributions - one due to the oscillations of the elastic membrane, the other due to the hits of the nut against the balloon - and present models to describe the frequency's dependence on the main parameters of the system. We analysed the influence of several parameters such as the balloon's geometry, the nut's geometry and its mass.
\end{abstract}

\section{Introduction}

The study of spherical elastic shells probably started at the end of the 19th century with the theoretical studies of Lamb [1,2]. Several refined theoretical studies have focused on determining the eigenfrequencies (and the amplitude) of such systems [3,4]. Works on spherical shells have also tackled the problem numerically $[5,6]$ and more complex work has appeared such as the study of instabilities of expanding and contracting shells [7].

Using large elastic balloons and the reflection of a laser on the surface of its membrane, Kuo et al. [8] observed these frequencies experimentally. Using an improved model from the previous literature, they were able to show good agreement between theory and experiments.

Nonetheless these previous studies are in majority theoretical and the experimental studies only focus on the frequency response of the vibrations of the membrane generated by a precisely controlled excitation. Hence, the study of more complex phenomenon is left open.

In this article, using different types of nuts (and coins) made to rotate inside an elastic rubber balloon, we investigate both the frequency content and the amplitude of the sound produced by the internal periodical excitation (produced by the rolling of a nut).

First, we present the different experimental methods and the theoretical models for the frequency components. Then, we report our experimental results and finally discuss the agreement between those experiments and our predictions.

\footnotetext{
* e-mail: amaury.barral@polytechnique.edu

e-mail: quentin.louis@polytechnique.edu
}

\section{Experimental method}

\subsection{Experimental setup for sound capture and analysis}

In most of the experiments, hex nuts were used. However, we also investigated nuts with several other shapes and report results for coins as well.

A nut of side length $l$ (with a total of $N$ sides), width $e$ and mass $m$ (see Fig. 1a) is inserted in a new (non-inflated before) transparent commercial rubber balloon (Nuolux 12 inches white latex balloons). The balloon is inflated by a human operator and tightly closed by pinching the nozzle, allowing it to be reopened and inflated further. Once inflated, the new dimensions of the balloon are measured on ruler-calibrated pictures. As in Figure $1 \mathrm{~b}$ the human operator then gives the balloon a circular translation movement, inducing the swirling of the nut inside the balloon.

The sound is recorded using a calibrated-response microphone (Shure SM57) at a fixed distance from the center of the balloon, facing the side of the balloon, the motion of the nut is captured by a high-speed camera (Photron FASTCAM SA at $3000 \mathrm{fps)}$ and a regular reflex camera (Canon EOS 700D) is used for pictures and $30 \mathrm{fps}$ videos. A schematic view of the experimental setup appears in Figure 1c.

The sound is analysed via its Fourier transform from Audacity 2.2.1 with size 65536 using a Hanning window, on samples of a few seconds. Temporal Fourier Transform and Amplitude calculations are also performed using Python. A typical Fourier transform and its integrated PSD (Power Spectral Density) are shown in Figure 2. Sound occurs through all the spectrum but 
(a)

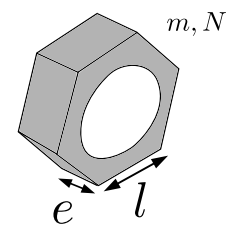

(b)

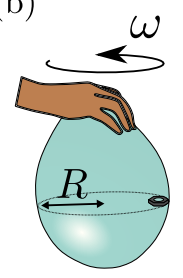

(c)

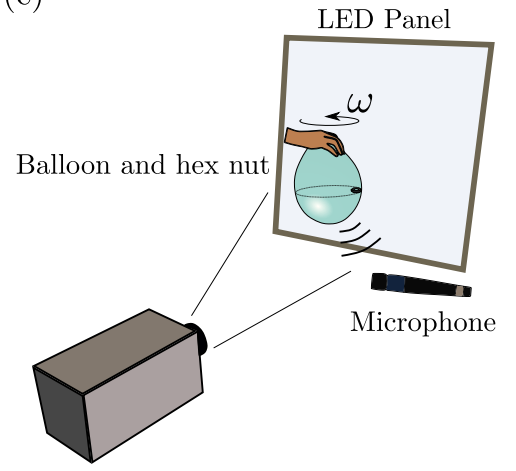

High speed camera

Fig. 1. Experimental setup and nomenclature. (a) Sketch of the hex nuts used in experiments. A nut is characterised by its number of sides $N$, it's side length $l$, its width $e$ and its mass $m$, (b) Close sketch of the balloon, characterised by its radius $R$ and its rotating speed $\omega$. (c) Sketch of the experimental setup.

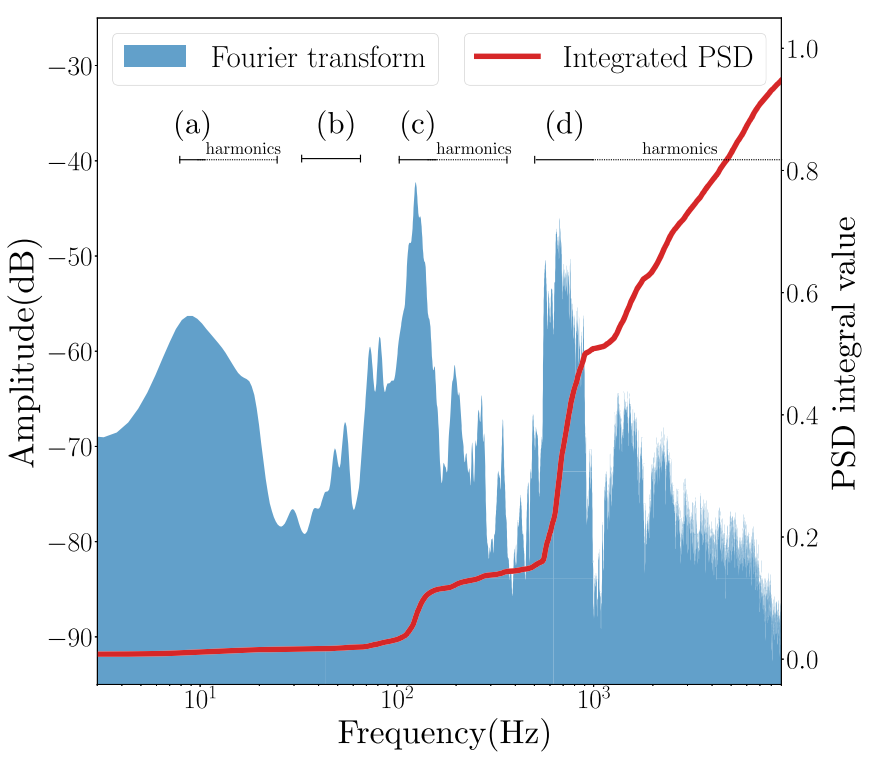

Fig. 2. Typical Fourier transform. In this case, the experimental conditions were: $N=6, m=0.3 \mathrm{~g}, l=2.7 \mathrm{~mm}, e=2.4 \mathrm{~mm}$, $f_{\text {rot }}=3.7 \mathrm{~Hz}$ and $R=9.8 \mathrm{~cm}$. Four identifiable zones (a), (b), (c), (d). Only (c) and (d) are audible as showed by the PSD (Power Spectral Density) integral. (a) Is the macroscopic sound produced by the balloon pushing air during its movement. (b) Is the sound components ranging from 30 to $80 \mathrm{~Hz}$. (c) Is the first eigenfrequency of the membrane. (d) Is the frequency produced by the nut rolling and hitting the membrane. Harmonics are also produced.

the higher frequencies are the only ones audible (in most cases), which the PSD integral confirms as its slope changes abruptly. The sound can be divided into four parts: two low frequency parts $(3-6 \mathrm{~Hz}$ and $40-80 \mathrm{~Hz})$, a medium frequency part $(100-200 \mathrm{~Hz})$ and a higher

frequency part that is much more variable depending on the characteristics of the nut $(200-2000 \mathrm{~Hz})$.

\subsection{Assumptions of our model}

\subsubsection{Influence of gravity}

Changes in the nut's height during its trajectory lead to changes in its velocity and thus in the sound we hear. Assuming this change to be $10 \%$ (from the horizontal plane) of the balloon's radius and with an initial speed of 1-3 $\mathrm{ms}^{-1}$, taking the condition $\Delta v / v<0.1$ where $v$ is the speed of the nut at the center height of the balloon and $\Delta v$ is the difference between the speed of the nut at the highest point of the trajectory and at the middle of the balloon, we get $g R / v^{2}<1$ where $R$ is the radius of the balloon and $g$ is the acceleration of gravity, which yields $R<$ $20 \mathrm{~cm}$ (experimentally $R$ varies between $3 \mathrm{~cm}$ and $15 \mathrm{~cm}$ ).

We can therefore safely neglect the influence of gravity in our experiments.

It is useful to note that even if gravity had a non negligible influence, it would just broaden the spectrum of frequencies around the gravity-less value, which only increases error bars.

\subsubsection{Influence of inertial forces}

Balloons are not perfectly spherical, they are rather pear shaped. This change in their radius induces a change in the inertial energy of the nut $E=\frac{1}{2} m \omega^{2} R^{2}$. Taking the condition $\Delta v / v<0.05$ gives $\Delta R<0.05 v^{2} / R \omega^{2}$ where $R$ is the radius of the balloon at the center of the balloon, $\Delta R$ the change in radius during the trajectory, $v$ the speed of the nut, $\omega=2 \pi f_{\text {rot }}$ the rotation pulsation of the nut, which for our typical setups gives $\Delta R<6 \mathrm{~cm}$ across the trajectory of the nut (experimentally $\Delta R$ is of the order of a few $\mathrm{cm}$ at most).

We can therefore safely neglect the influence of uncontrolled changes in the inertial force in our experiments.

Even if changes in the inertial force had a non negligible influence, it would be equivalent to greater uncertainties in the measure of the balloon's radius.

\subsubsection{Influence of the Doppler effect}

The relative frequency shift induced by the Doppler effect is $\Delta f / f=v / c$ where $v$ is the speed of the nut and $c$ is the speed of sound in air. We consider it negligible for $\Delta f / f<0.01$ which gives us $v<3 \mathrm{~ms}^{-1}$. Typical speed ranges in our experiments are $1-3 \mathrm{~ms}^{-1}$.

We can therefore safely neglect the influence of the Doppler effect in our experiments.

\subsubsection{Influence of friction}

Experiments show that the nut does not slide on the membrane of the balloon, and that changing the friction coefficient by adding oil (which dramatically reduces friction) has no significant influence on the sound emitted, which leads us to affirm that friction is not an important parameter in this problem. This allows us to use a no-slip model to predict the primary frequency. 


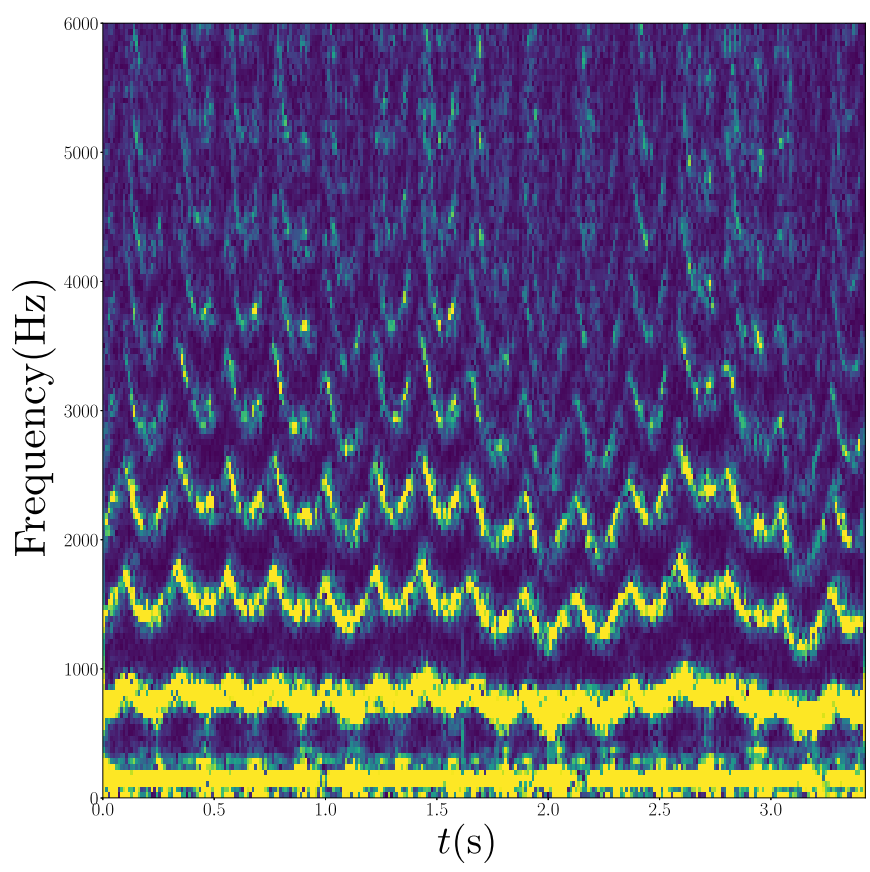

Fig. 3. Spectrogram of a typical audio record of 3 seconds. Experimental conditions are identical to Figure 2. Frequency components are plotted as a function of time. The ladder of intensity goes from dark blue (no frequency component) to yellow (important frequency component). The amplitude modulation is represented by the alternation of bright (yellow) and dark zones. Oscillations of the harmonics of the main frequency $(\sim 800 \mathrm{~Hz})$ with the same periodicity as the amplitude modulation are also visible.

\subsection{Rotational frequency of the nut}

Within our range of speeds and dimensions, the frequency at which the nut rotates is equal to the frequency at which the amplitude is modulated.

Since the amplitude of the sound received depends on the distance between the nut and the microphone (which varies significantly during the experiments as the nut goes from one side of the balloon to the other) we observe a modulation frequency on the spectrograms. The periodicity of the spectrogram in Figure 3. is theoretically equal to the rotation frequency of the nut. This is experimentally checked using a camera.

\subsection{Measurement of elastic properties}

The elastic properties (the Young's Modulus and Poisson's ratio) of commercial rubber balloons are not specified and need to be determined experimentally. To do so, we cut bands of known dimensions from new balloons and stretch them while measuring the force required to do so. We also measure the thickness/width of the band during the stretching experiment. The Young's modulus of a band of length $l$ is defined by $E=\sigma / \epsilon$ where $\sigma$ is the stress (force/surface) applied and $\epsilon=\Delta l / l$ is the relative elongation of the band. Poisson's ratio $\nu$ is defined by the amount of transverse expansion divided by the amount of axial contraction.
In our experimental conditions, we are not in the elastic regime on the whole strain curve. However, once the balloon is inflated, the slope of the strain curve around the inflation deformation is locally constant, and we can define a local Young's modulus for a given deformation. The additional deformations of the inflated balloon during the experiments, either due to the hex nut pushing on the membrane or to waves travelling in the membrane, are small enough to justify that this approximation holds.

\subsection{Measurement of the main sound frequency - nut hits on the membrane}

The primary sound's frequency produced is measured by taking the frequency corresponding to the main component in the spectrogram.

\subsection{Measurement secondary sound frequency}

In experiments appear a rather constant frequency that can be heard in some remote cases between 100 and $200 \mathrm{~Hz}$. We measure this frequency by taking the highest amplitude component between 100 and $200 \mathrm{~Hz}$ in the spectrogram.

\subsection{Measurement of the amplitude amplification}

Due to its shape and oscillating properties we expect the balloon to act as a sound box, amplifying the sound produced by the nut.

To measure this amplification we measure the sound produced by a piezoelectric transducer in the air at a given frequency, then compare it to the sound produced when a balloon is attached to the transducer under the same conditions. The difference in amplitude between the two experiments gives us the amplification for that frequency. We then obtain a continuous graph by sweeping between frequencies.

The amplitude considered here is the total amplitude of the sound, with all its frequencies.

\subsection{Measurement of the amplitude of the main sound}

Since the primary sound is the one with the highest fundamental frequency and does not overlap with other sound contributions in the studied frequency range, we extract its amplitude from the Fourier transform by integrating the peaks from $80 \%$ of the fundamental frequency (to account for the peaks width) to infinity.

\section{Models}

\subsection{Predicted main sound frequency - nut hits on the membrane}

The primary sound produced is due to the nut deforming the membrane and creating a pressure wave at a given frequency. The frequency at which a nut of side length $l$ rolling without friction at a speed $v$ hits the balloon is $f_{\text {pred }}=v / l$. Harmonics are also generated by the deformation. 


\subsection{Predicted secondary sound frequency - membrane vibration}

A rubber balloon is an elastic medium with periodic boundary conditions, we thus expect it to have specific eigenfrequencies at which the membrane is going to oscillate and create a sound.

Following Baker [3] we model our balloon as a simple spherical elastic shell. Its eigenfrequencies are given by

$$
f_{n}^{2}=\frac{\alpha \pm \sqrt{\alpha^{2}-4\left(1-\nu^{2}\right)[n(n+1)-2]}}{2 G}
$$

where $\alpha=n(n+1)+1+3 \nu, G=\frac{\left(1-\nu^{2}\right) \rho R^{2}}{E} . R$ is the radius of the balloon, $E, \nu$ are its Young's modulus and Poisson's ratio respectively, and $\rho$ is the rubber's density. The \pm sign yields two branches of eigenfrequencies, thereafter named lower branch and upper branch.

More simply, we can derive the dependency of the frequency in $1 / R$ with a simple rope model. Assimilate the balloon sphere to a rope of length $L=2 \pi R$ with periodic boundary conditions. The wavelengths $\lambda$ will be quantified, proportional to $L / n$ where $n \in \mathrm{Z}$. Therefore, $f=c_{e l} / \lambda \propto 1 / R$ where $c_{e l}$ denotes the speed of sound in the elastic material.

\section{Results}

\subsection{Influence of solid friction}

The movement of the nut on the membrane is circular as Figure 4a shows that it corners' motion is sinusoidal. The chronophotography in Figure $4 \mathrm{~b}$ confirms this rolling behaviour: when the nut topples over, its corner in contact with the membrane does not slide.

\subsection{Rotational frequency of the nut}

The frequency of the modulation of amplitude is plotted against the rotational frequency of the nut (captured by video) in Figure 5.

\subsection{Elastic properties}

Traction curves such as in Figure 6 give us an estimate on the Young's modulus of our balloons of 1-3 Mpa. Regular measurements of the thickness/width of the membrane give a Poisson's coefficient between 0.4 and 0.5 , within expected ranges (theoretical value 0.5 for an elastic medium).

\subsection{Main frequency - nut hits on membrane}

We determine the primary frequency of the sound as a function of the radius of the balloon, the side length of the nut and its rotation frequency inside the balloon. The theoretical frequency is plotted against the experimental frequency in Figure 7.
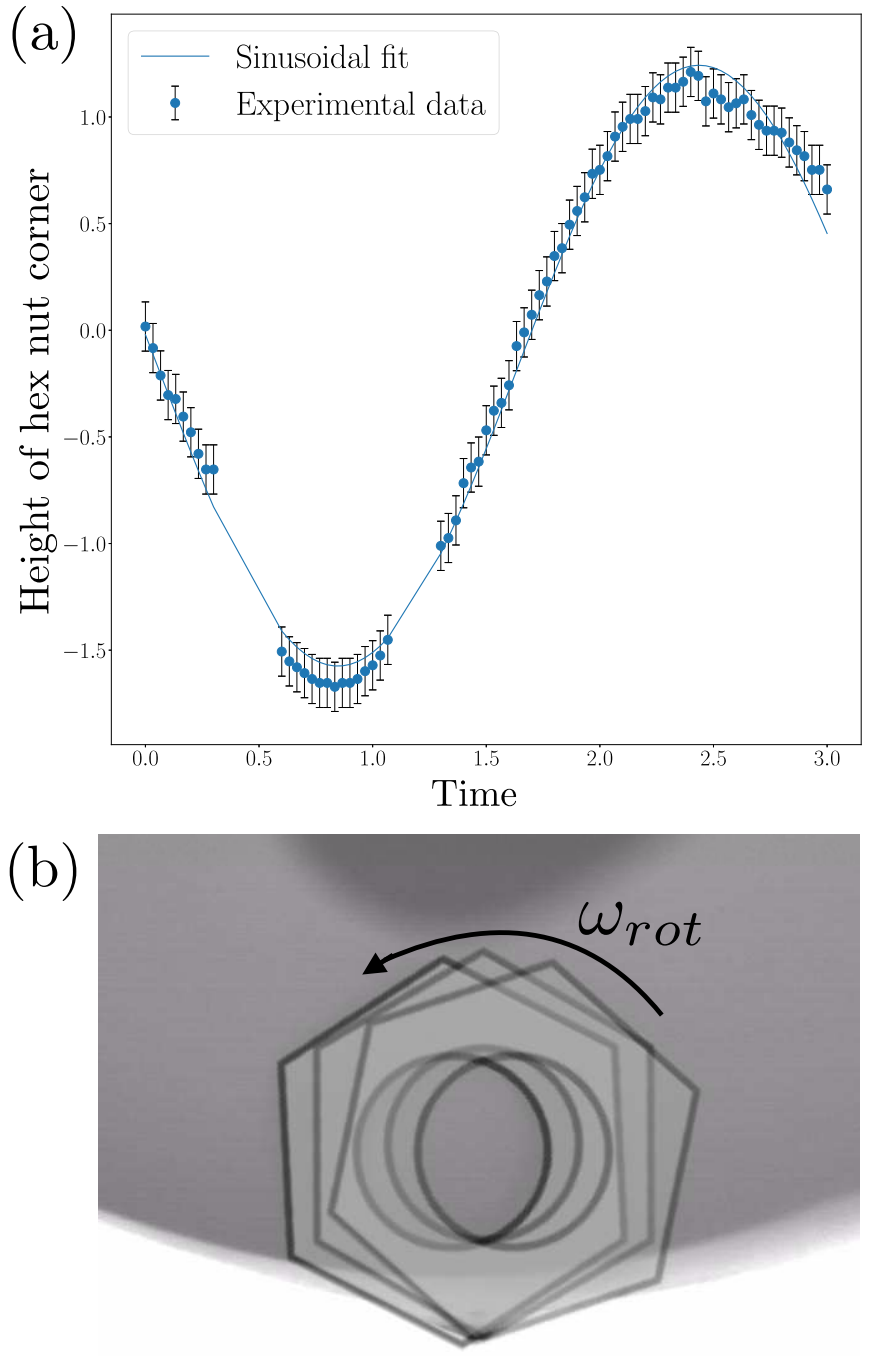

Fig. 4. (a) Experimental data of the height of a corner of the nut during one rotation of the nut extracted using Tracker (software). A sinusoidal fit is performed on the experimental data and plotted in solid line. (b) Superposition of three images of the nut rolling on the membrane taken with a high-speed camera at 3000 fps. For visualisation purposes the hex nut is redrawn.

We found no influence of the mass and width of the nut on this frequency.

\subsection{Secondary frequency - membrane vibration}

Using a high-speed camera at $3000 \mathrm{fps}$, we observe vibrations on the surface of the membrane. The absence of either visible nodal points or asymmetrical motion indicates that the fundamental mode is dominant as described in the model we used [3].

We determine the frequency of the sound produced by the membrane in function of the radius of the balloon. The experimental frequency is plotted against the inverse of the radius in Figure 7.

We found no influence of the mass, length, width and rotation frequency of the nut on the frequency. 


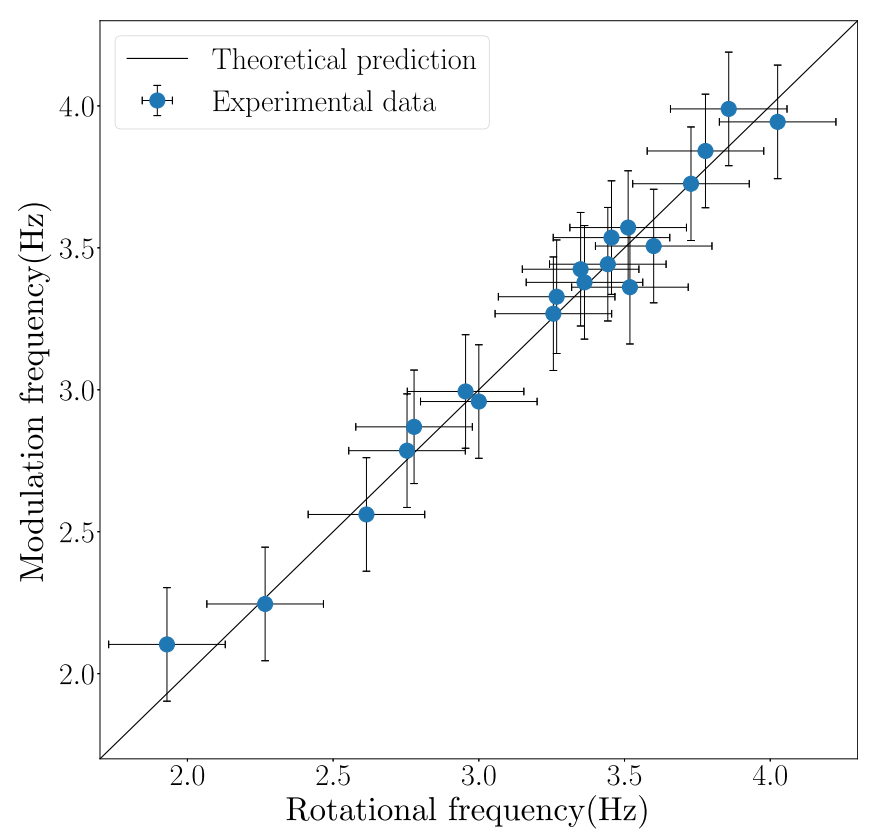

Fig. 5. Amplitude modulation frequency plotted against the macroscopic rotational frequency of the nut. The frequency was averaged over a period of 10 modulations for each experiment. The macroscopic rotational frequency was derived from videos of the balloon taken at $30 \mathrm{fps}$, using a regular camera.

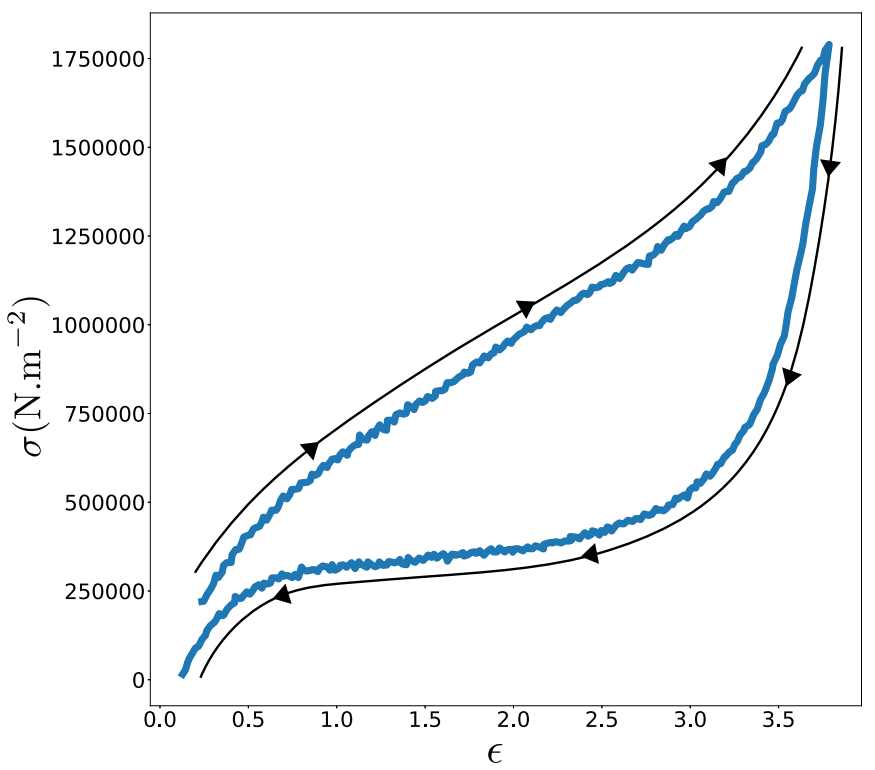

Fig. 6. Traction curve of an elastic rubber balloon. The stress $\sigma$ is plotted as a function of the relative elongation of the band $\epsilon$. Black arrows show the path of the deformation. Their is a non-linear stretching deformation (arrows upwards). The loosening (arrows downwards) does not follow the initial curve, a characteristic feature of the plastic domain.

\subsection{Amplitude amplification}

We plot the amplification due to the sound-box-like properties of the balloon against the frequency in Figure 8. The amplification is calculated by subtracting the amplitude
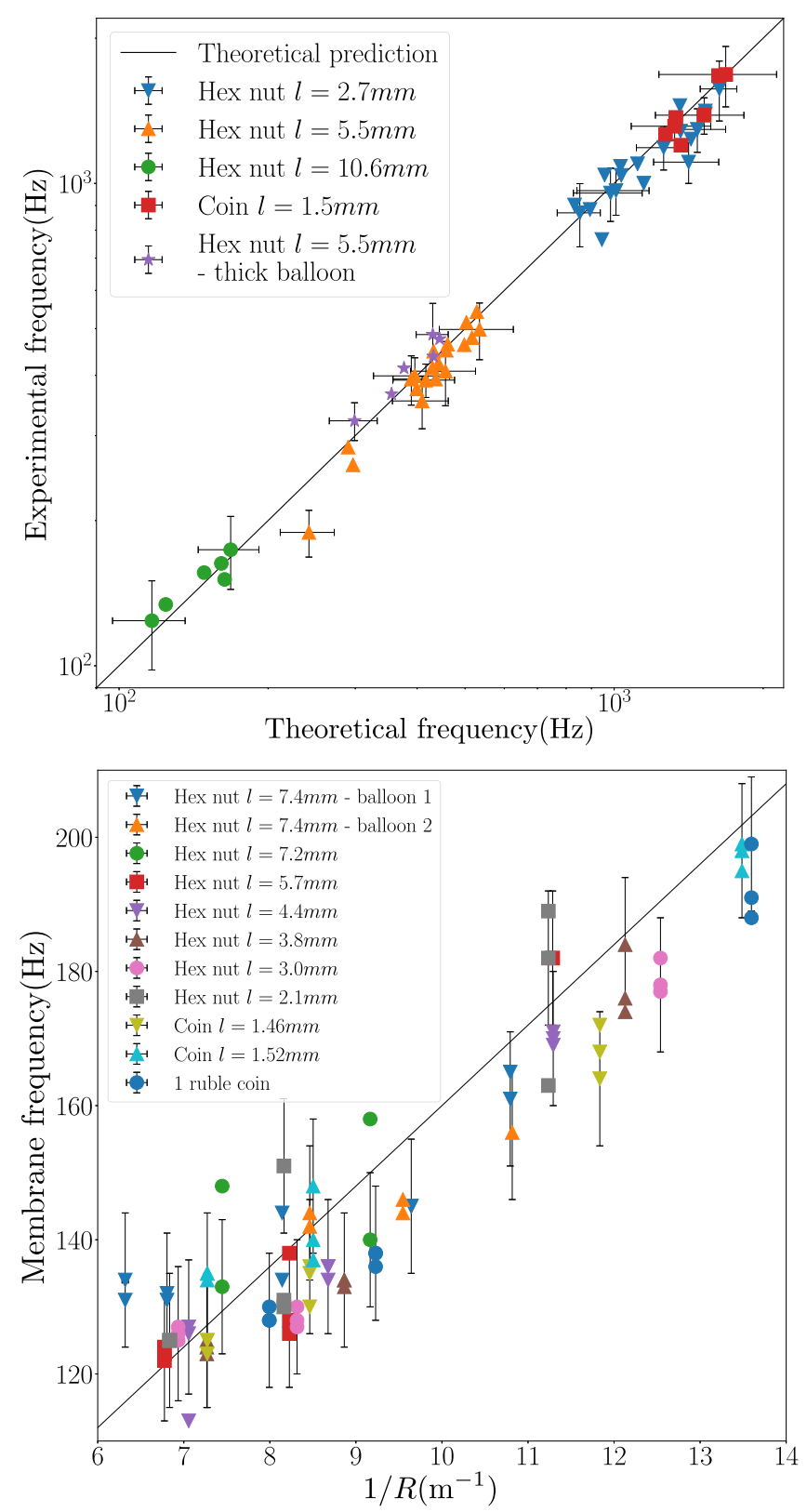

Fig. 7. (Top) Theoretical frequency of the sound plotted against the experimental main sound frequency. A wide range of balloon radius $(3-15 \mathrm{~cm})$ and different nuts/coins (see legend) were studied. Frequencies ranging from $100 \mathrm{~Hz}$ to little less than $2000 \mathrm{~Hz}$ were reached. The theoretical prediction is plotted as a black line. (Bottom) Lowest vibrational frequency of the balloon membrane plotted as a function of $1 / R$. A wide range of balloon radius $(3-15 \mathrm{~cm})$ and different nuts/coins (see legend) were studied. Frequencies from $100 \mathrm{~Hz}$ to $200 \mathrm{~Hz}$ were reached.

of the sound produced by the transducer alone from the sound of the transducer stuck to the balloon.

\subsection{Amplitude of the main sound}

In our experiments, we used the same type of balloon but varied its radius $R$ as well as the parameters of the nut (mass $m$, width $e$ and side length $l$ ). 


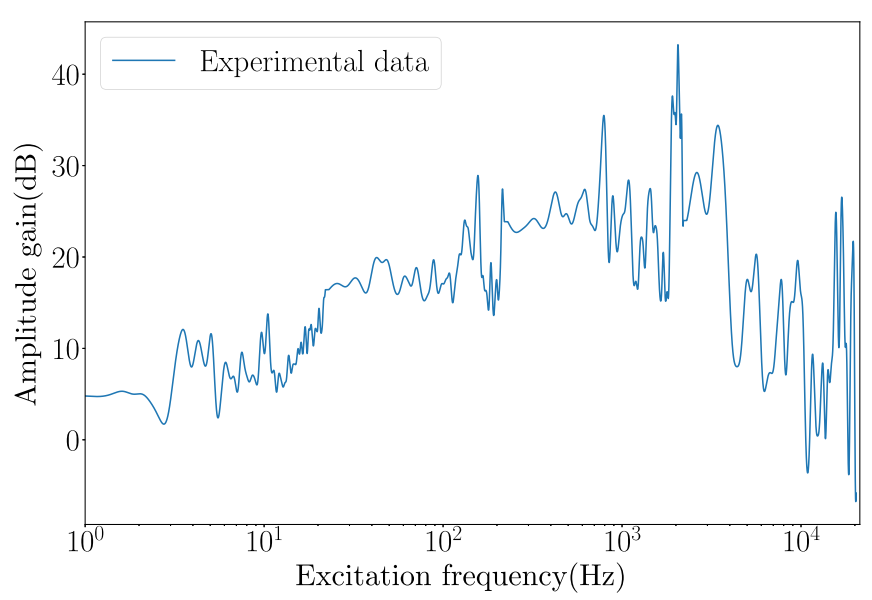

Fig. 8. Amplification (in $\mathrm{dB}$ ) is plotted as function of the frequency at which a piezoelectric transducer excites the membrane. Amplification was derived from the difference of amplitude between the transducer vibrating alone and its action as it was put on the balloon. For convenience, a sweep of frequencies was performed. The distance to the microphone remains constant between the two sound samples.

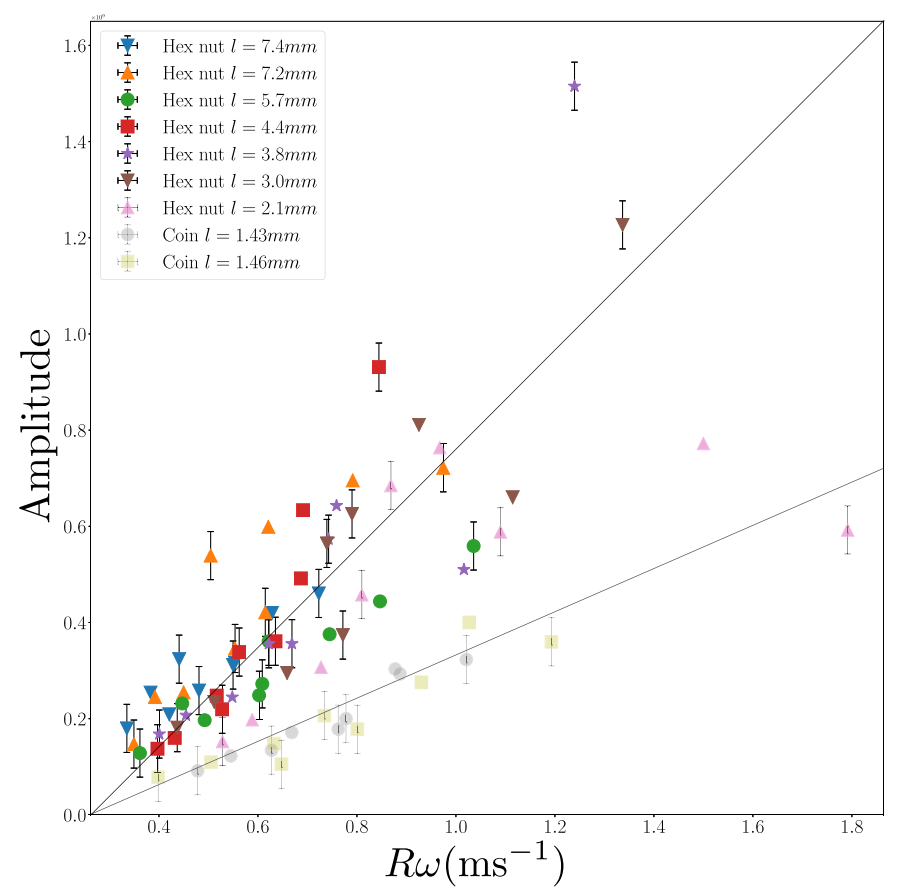

Fig. 9. The amplitude $A$ plotted as a function of $R \omega$ for the same type of nuts, same rotation speeds and same balloon radii as above. Linear regressions are plotted for each type of nuts. Coin data points are plotted with low opacity due to deviation from the regression coefficient of the hex nuts.

We plot the amplitude $A$ as a function of the translation velocity of the nut $R \omega$ in Figure 9.

We also plot the linear fits for all types of nuts.

\section{Discussion}

\subsection{Rotation frequency of the nut}

As shown in Figure 5, experiments attest that in our range of speeds and radii, the rotation frequency of the nut can be approximated with a good precision by the amplitude modulation's frequency.

In other experiments, we take the amplitude modulation's frequency as the rotational frequency, as it is significantly less time-consuming and experimentally constraining than tracking the nut on a video.

\subsection{Main sound frequency - nut hits on membrane}

Our model predicts a frequency varying as $f_{\text {pred }}=v / l$ using aforementioned notations. Assuming constant speed over the perimeter of the balloon this yields $f_{\text {pred }}=$ $(2 \pi R / l) \times f_{\text {rot }}$ where $f_{\text {rot }}$ is the rotation frequency of the nut.

As shown at the top of Figure 7, experiments show a very good agreement between the predicts and experimental frequencies. We conclude that the main audible sound is indeed produced by the shocks of the nut's sides on the membrane of the balloon at a given frequency.

\subsection{Secondary sound frequency - membrane vibration}

As shown at the bottom of Figure 7, experimental frequencies are in good qualitative agreement with the predicted eigenfrequencies in [3] for both the lower and upper branch. The lower branch, very tightly packed, coincides with the main peak of the membrane's sound. The upper branch, more diluted, is harder to analyze, and corresponds to eigenmodes harder to excite with free boundary conditions.

Baker as well as our simple rope toy model predict a dependence of the eigenfrequencies in $f \propto 1 / R$, which is experimentally verified and justifies our model.

\subsection{Amplitude amplification}

As shown in Figure 8, experiments show a roughly constant amplification over the audible range of $+20 \mathrm{~dB}$ in the range of a $100 \mathrm{~Hz}$ to around $4000 \mathrm{~Hz}$, which explains why we can hear the sound of the nut.

\subsection{Amplitude of the primary sound}

Results in Figure 9 show that for hex nuts, the amplitude of the sound produced collapses well under $R \omega$ (the translation velocity of the nut). Although we performed some in depth modelling of the amplitude, we were not able to provide any satisfactory account of experimental results.

A lower linear coefficient for coins as well as for the smallest nut at high speed suggest some saturation phenomena of the amplitude that could be due to the saturation of the deformation of the membrane (due to the smallness of the those nut's sides). 


\section{Dead ends and possible improvements}

\subsection{Higher order eigenfrequencies}

Measuring higher order eigenfrequencies proves a very difficult task using Fourier transforms. Due to their smaller amplitudes they tend to overlap with other less significant signals or with one another, especially in the lower branch. A setup based on laser measurements as done by Kuo [8] would be more suited for such experiments.

\subsection{Mechanical setup}

Doing the experiments by hand induces non negligible incertitudes. We also used a mechanized setup via a lab shaker but did not present any such results here. Indeed, that setup has two major drawbacks: first of all it creates a nontrivial background noise, which can not easily be removed by simple algorithms, and masks most membrane frequencies. The other issue is that while using a mechanized setup we encountered a new phenomena of frequency modulation due to the forced excitations, which hinders our experiments. We believe this phenomena to occur the following way: to a given excitation frequency is associated a stable orbit in the balloon. If the nut is not initially on this orbit, it will move towards it due to the normal reaction on the balloon's surface. Due to inertia, it will however not stop at the stable orbit but overshoot it. This induces an up/down motion of the rotation plane, and thus a modulation of the sound. This is backed up by experimental data but we do not discuss it further here.

\subsection{Influence of gas}

Our model does not take into account the inner and outer gas. This has been studied by Kuo [8] but requires experimental data we do not have (such as the pressure difference across the membrane). We expect the gas to have no significant influence on the main sound's frequency, save for the heavy gases which could leads to non negligible dampening of the oscillations and thus a change in the harmonics' amplitude and frequency. We do expect a dependency of the membrane's eigenfrequencies with the gas's density.

\subsection{Unexplained frequencies}

During most experiments we consistently observe between 30 and $80 \mathrm{~Hz}$ a block of inaudible frequencies that seems relatively unaffected by experimental parameters. A quick analysis did not permit to determine whether this was only due to noise. We believe this block might be due to fine details on the nut's geometry but do not discuss it further here.

\subsection{Theoretical model for the amplitude}

Although results show an interesting collapse of the data of amplitude with $R \omega$ dependency and despite a thorough research to provide a theoretical explanation of this dependency, we did not manage to provide any convincing proof. The issue residing in the large range of modelling hypotheses one can make which would require more experimental data with a wider range of different nuts to be tested.

\section{Conclusion}

In this article we have investigated the sound produced by a nut when it is made to roll inside a balloon, both experimentally and theoretically. After discussing the behavior of the nut on the membrane and explaining the amplitude modulation that one can hear when listening to the sound emitted we have studied the two main components of the sound emitted: membrane vibrations and the impact of the nut on the membrane. Regarding the membrane oscillations we showed experimentally that the frequency of the lower branch varies as the inverse of the radius of the balloon. Regarding the impact of the nut we derived the theoretical frequency of the sound that correlates very well with the main frequency component measured in the experiments. On the amplitude of the sound, we studied the effect of the balloon acting as a sound box and experimentally showed that in the range of frequencies at which the nut rolls the amplification of the sound is around $20 \mathrm{~dB}$. For nuts, we experimentally showed a linear dependency of the amplitude of the sound emitted on the speed of nut.

This work is based on a problem proposed in the 2018 edition of the International Physicists' Tournament (IPT). We are grateful to Christophe Clanet, from the LadHyX, Guilhem Gallot from the LOB and Fabian Cadiz from the PMC at Ecole polytechnique for their fruitful suggestions and corrections brought to the core of this article. We also thank the teams from ParisSud at the French Physicists' Tournament (FPT) and the teams of Switzerland and Brazil at the IPT for the intense discussions we had in order to solve this problem.

\section{Author contribution statement}

Amaury Barral and Quentin Louis have contributed equally to this work.

\section{References}

1. H. Lamb, On the vibrations of an elastic sphere, Proc. Lond. Math. Soc. 1, 189-212 (1881)

2. H. Lamb, On the vibrations of a spherical shell, Pro. Lond. Math. Soc. 1, 50-56 (1882)

3. W.E. Baker, Axisymmetric modes of vibration of thin spherical shell, J. Acoust. Soc. Am. 33, 1749-1758 (1961)

4. A. Silbiger, Nonaxisymmetric modes of vibration of thin spherical shells, J. Acoust. Soc. Am. 34, 862 (1962)

5. A.R. Robinson, M.S. Zarghamee, A numerical method for analysis of free vibration of spherical shells, AIAA J. 5, 1256-1261 (1967) 
6. S.K. Panda, B.N. Singh, Nonlinear free vibration of spherical shell panel using higher order shear deformation theory-a finite element approach, Int. J. Pres. Vessels Pip. 86, 373-383 (2009)
7. A. Goriely, M.B. Amar, Differential growth and instability in elastic shells, Phys. Rev. Lett. 94, 198103 (2005)

8. K.A. Kuo et al., Small oscillations of a pressurized, elastic, spherical shell: model and experiments, J. Sound Vib. 359, 168-178 (2015)

Cite this article as: Amaury Barral, Quentin Louis, Clément Brochet, Julie André, Anthony Guillen, Fang Goh, Angel Prieto, Thibault Guillet. Sound of a nut rolling inside an elastic rubber balloon, Emergent Scientist 3, 6 (2019) 